\title{
Hilfsmittel und Verfahren für zellbasiertes high content imaging
}

DANIEL F. GILBERT, OLIVER FRIEDRICH, CHRISTIAN LESKO, KRISTIN HARTMANN LEHRSTUHL FÜR MEDIZINISCHE BIOTECHNOLOGIE,

UNIVERSITÄT ERLANGEN-NÜRNBERG

\section{Cell-based high content imaging has become a versatile and powerful} method for studying e. g. cellular viability, morphology or physiology. However, high content imaging and its various applications require complex as well as expensive hardware and consumables, limiting its utility and usability. To broaden the applicability of cell-based high content imaging, we have developed tools and assaying techniques being exemplary portrayed in this article.

DOI: $10.1007 / \mathrm{s} 12268-021-1542-3$

(C) Die Autoren 2021

High content imaging kommt in ganz unterschiedlichen Forschungsdisziplinen für eine Vielzahl von wissenschaftlichen Fragestellungen zum Einsatz. Am Lehrstuhl für Medizinische Biotechnologie der FriedrichAlexander-Universität Erlangen-Nürnberg (FAU) in Erlangen nutzen wir high content imaging u. a. für zellbasierte Experimente, z. B. um mit Antikörpern die räumliche und zeitliche Verteilung von Proteinen zu unter- suchen, um die Auswirkung von Testsubstanzen auf die zelluläre Gesundheit und Morphologie zu studieren, oder um neuartige Wirkstoffe zu identifizieren, die sich auf die Funktion von Ionenkanälen und damit die zelluläre Physiologie auswirken. Bei diesen Untersuchungen setzen wir high content imaging-Mikroskope ein, die für spezifische Fragestellungen mit spezieller Hardware ausgestattet sind. So erfordern z. B. immun- zytochemische Untersuchungen mit fluoreszenzmarkierten Antikörpern die Verwendung von Bauteilen zur Fluoreszenzanregung und -detektion und beim Wirkstoffscreening kommen Pipettiersysteme zum Einsatz, die es ermöglichen, große Substanzbibliotheken automatisiert zu analysieren. Diese spezielle Hardware ist zwar einerseits teuer in der Anschaffung und erfordert speziell geschultes Personal für die Anwendung und Instandhaltung, andererseits ermöglicht sie ein breites Spektrum von high content imaging-Anwendungen und ist daher für einen Großteil von wissenschaftlichen Fragestellungen unabdingbar.

Trotzdem: Die hohen Kosten für high content imaging-Geräte und der implementierten Zusatzhardware limitieren die Verfügbarkeit für kleinere bzw. finanziell weniger gut ausgestattete Labore, insbesondere in Ländern mit niedrigem und mittlerem Einkommen. Fragestellungen oder Projekte, die nur mit spezieller und mit Zusatzhardware ausgestatteter high content imaging-Technologie zu realisieren sind, können von diesen Laboren daher häufig nicht bearbeitet werden. Hinzu kommen kostenintensive Verbrauchsmittel
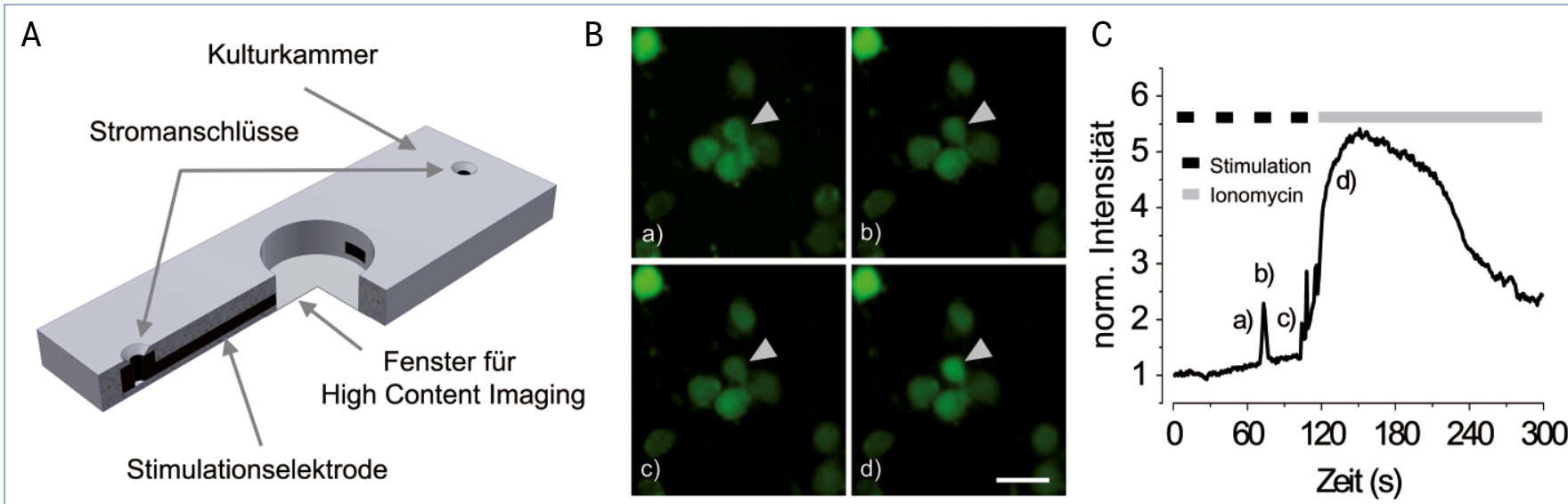

$\Delta$ Abb. 1: 3D gedruckte wiederverwendbare Kulturkammer mit integrierten Elektroden für die elektrische Stimulation und parallele mikroskopische Analyse. A, 3D-Modell der Kammer. B, Fluoreszenzaufnahme Fluo-4-beladener MED 17.11-Zellen zu verschiedenen Zeitpunkten während des Stimulationsexperiments. Größenmarker: $100 \mu \mathrm{m}$. Der Pfeil markiert eine Zelle, die infolge der Stimulation variierende Fluoreszenzintensitäten aufwies. C, Intensitätsverlauf der markierten Zelle aus B. Nach der wiederholten Stimulation wurden die Zellen mit dem Calcium-lonophor lonomczin behandelt, um ein maximales Fluo-4-Intensitätssignal auszulösen und zu demonstrieren, dass es sich bei den Intensitätsschwankungen um Calcium-abhängige Signale handelt. Modifiziert aus [1]. 


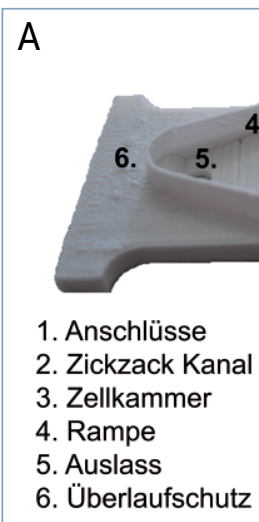

1. Anschlüsse

2. Zickzack Kanal

4. Rampe

6. Überlaufschutz
B

a)

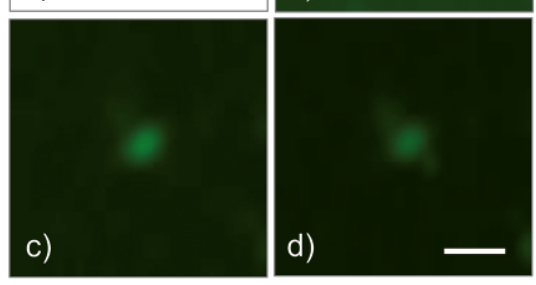

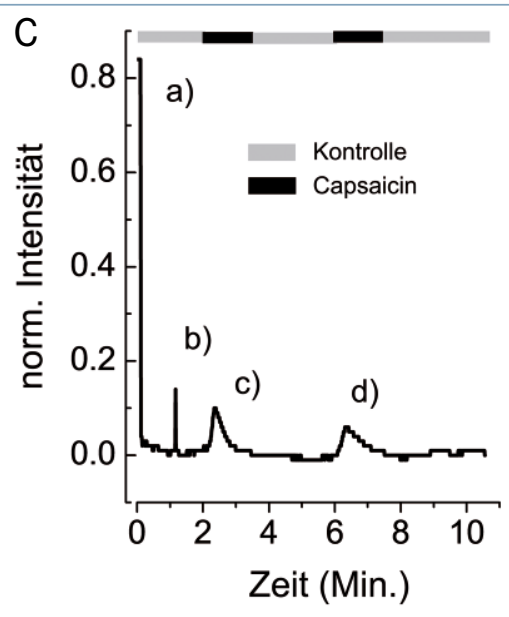

$\Delta$ Abb. 2: 3D gedruckter Lab-Chip zur chemischen Stimulation und parallelen high content imaging-basierten Analyse. A, 3D gedruckte Vorrichtung mit Einlässen für Kontroll- und Testlösung (1.), Zickzack-Kanal zum Mischen der beiden Lösungen (2.), Zellkammer für die Mikroskopie (3.), Rampe zum Ableiten der Messlösung Richtung Auslass (4.), Auslass mit bodenseitigem Anschluss (5.) und Überlaufschutz (6.). Das Bild unterhalb des Chips zeigt die Struktur des Kanalsystems. B, Fluoreszenzaufnahme Fluo-4-beladener HEK293-Zellen zu verschiedenen Zeitpunkten während des Experiments. Durch eine Luftblase in der Zellkammer bei Experimentbeginn ist das Bild in a) gesättigt und weiß dargestellt Größenmarker: $50 \mu \mathrm{m}$. C, Intensitätsverlauf der mit Kontroll- und Testlösung perfundierten Zelle aus B. Modifiziert aus [2].

für die Durchführung zellbasierter imagingExperimente, wie spezielle Kulturschalen, die es z. B. erlauben, Zellen zu reizen und parallel mikroskopisch abzulichten. Bei diesen - als funktionelles imaging - bezeichneten Messungen werden Kulturzellen über einen Zeitraum von Sekunden, Minuten oder sogar Stunden kontinuierlich abgelichtet und währenddessen ein- oder mehrfach mechanisch, chemisch oder elektrisch stimuliert. Aus der Reaktion der Zellen bzw. verwendeter Biosensoren, wie z. B. Fluoreszenzmarker, lassen sich dann Rückschlüsse auf die Funktion intrazellulärer Proteine oder auch der zellulären Physiologie ziehen. Kostengünstige Verbrauchsmittel für solche Experimente sind kommerziell häufig nicht verfügbar. Um derartige Experimente trotz eines kleinen Forschungsbudgets durchführen zu können, ist ein Blick über den eigenen methodischen Tellerrand - in Richtung anderer Forschungs- oder Arbeitsfelder - sehr hilfreich.

\section{DIY-Werkzeuge für high content imaging}

Eine ungewöhnliche Situation erfordert außergewöhnliche Maßnahmen. Diese Aussage trifft insbesondere auf Forschungsfragen zu, die sich mit konventioneller Ausstattung nicht bearbeiten lassen, bzw. für die es keine Lösung von der Stange gibt. In diesem Fall kann ein Abstecher in die Maker-Szene helfen. Die Maker-Bewegung oder Do-ityourself(DIY)-Kultur arbeitet mit rapid prototyping-Technologien, wie additiver Fertigung
(3D-Druck), open-source MikrokontrollerBoards und Massenware aus dem Robotikund Elektronikfachhandel. Die Maker-Community bietet eine Vielzahl von Internetplattformen für den Austausch von Konstruktionsplänen für den 3D-Druck, von Schaltplänen zum Erstellen elektrischer Schaltkreise oder von Softwarecodes zum Programmieren von Mikrocomputern. Viele Universitäten weltweit verfügen mittlerweile über fab labs (fabrication laboratories) - offene Werkstätten, die es jedem interessierten Wissenschaftler ermöglichen, die eigene Konstruktionsidee in die Tat umzusetzen. Mithilfe dieser Maker-Tools und der offenen Werkstätten sind den vergangenen Jahren zunehmend Forschungsgeräte und -werkzeuge für das high content imaging entwickelt worden, die nicht nur kostengünstig sind, sondern auch jederzeit auf eine veränderte Fragestellung schnell und preiswert angepasst werden können.

\section{Kostengünstige Stimulations- kammern für high content imaging}

Ein Beispiel für ein DIY-Tool für high content imaging ist eine Kulturkammer für die elektrische Stimulation von Zellen, die wir am Lehrstuhl für Medizinische Biotechnologie an der FAU Erlangen-Nürnberg entwickelten. Die Vorrichtung fertigten wir mit einem dualextruder 3D-Drucker aus zwei unterschiedlichen Materialien - einem leitfähigen Filament für die Stimulationselektroden und einem elektrisch isolierenden Material sozusagen aus einem Guss. Zusätzlich statteten wir die Unterseite der gedruckten Kammer mit einem Sichtfenster aus Glas aus. Mit dieser Vorrichtung lassen sich Zellen, die in der Kammer wachsen, mit elektrischen Pulsen reizen und die Zellreaktion mithilfe spannungsabhängiger oder ionenselektiver Fluoreszenzfarbstoffe studieren. Da die Kammer die Abmessungen eines Standard-Objektträgers hat $(76 \times 26 \mathrm{~mm})$, kann sie mit jedem konventionellen high content-Fluoreszenzmikroskop zum Einsatz kommen. Aufgrund des geringen Bauvolumens und des einfachen Aufbaus ist die Kammer in kurzer Zeit gedruckt, in wenigen Stunden einsatzbereit und mit Materialkosten von weniger als einem Euro sehr kostengünstig. Eine Schemazeichnung der Kammer ist in Abbildung 1A gezeigt. Die Verwendbarkeit der Vorrichtung demonstrierten wir in einem proof-of-concept-Experiment mit Fluo-4-markierten MED17.11-Spinalganglienzellen. Die konditional immortalisierte Spinalganglienzelllinie exprimiert infolge einer chemisch induzierten Differenzierung neuronale Marker und eignet sich daher für die Untersuchung neuronaler Proteine und Eigenschaften. Für unser proof-of-conceptExperiment kultivierten wir differenzierte MED17.11-Zellen über Nacht in der Kammer und beluden sie am nächsten Tag mit dem Fluoreszenzfarbstoff Fluo-4 AM. Fluo-4 ist ein nicht-ratiometrischer Fluoreszenzindikator für die Untersuchung der intrazellulären Calciumkonzentration. Durch eine elektrische Stimulation depolarisiert das Membranpotenzial einer Nervenzelle, wodurch sich 

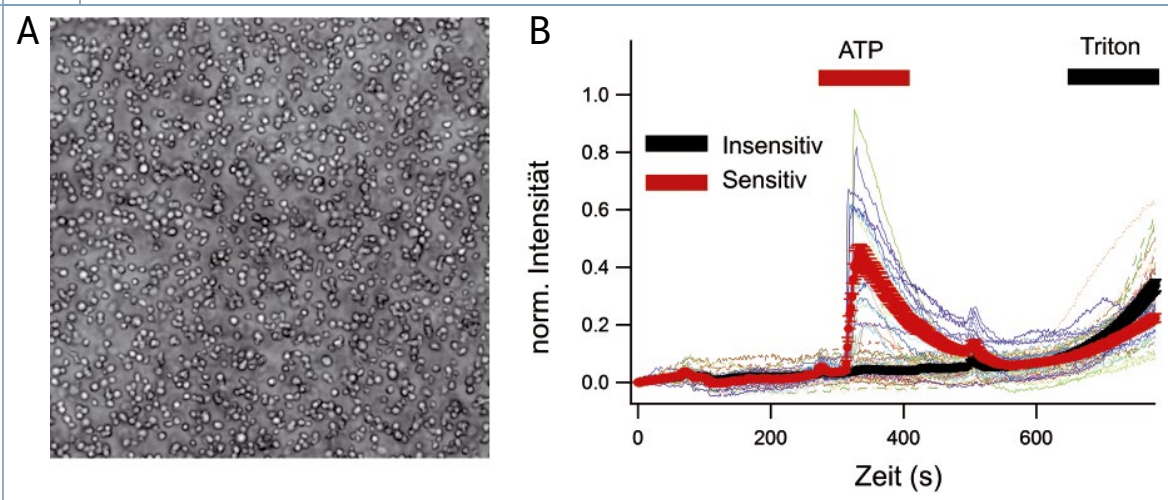

$\Delta$ Abb. 3: Methode für funktionelles high content imaging intrazellulärer Calciumantworten in Gelatine-immobilisierten nicht-adhärenten Suspensionszellen. A, Durchlichtaufnahme Gelatineimmobilisierter THP-Zellen. B, Normalisierte Einzelzell-basierte Antworten auf eine ATP- bzw. Triton-X-Exposition zur Untersuchung spezifischer bzw. unspezifischer Calciumsignale der Zellen aus A. In diesem Experiment haben etwa 30 Prozent aller Zellen (rot) spezifisch auf den ATP-Stimulus reagiert, was der Erwartung für den untersuchten Zelltyp entspricht und demonstriert, dass die Methode dazu geeignet ist, Calciumantworten menschlicher Blutzellen mittels high content imaging zu analysieren. Modifiziert aus [4].

spannungsgesteuerte Ionenkanäle in der Zellmembran öffnen und die Leitfähigkeit der Membran u. a. für Calcium erhöhen. Einströmende Calciumionen lassen die intrazelluläre Calciumkonzentration ansteigen. Dieser Anstieg resultiert in einer Zunahme der Fluo-4-Intensität, die wiederum durch high content imaging registriert werden kann. Abbildung 1B zeigt Fluo-4-beladene MED17.11-Zellen vor und während der elektrischen Stimulation. Der Intensitätsverlauf in Abbildung 1C lässt erkennen, dass sich die Fluoreszenzintensität im Verlauf des Experiments durch die elektrische Stimulation ändert. Für die Pulsgenerierung konstruierten wir zusätzlich mithilfe eines Arduino-Mikrokontroller-Boards einen kostengünstigen Stimulator. Um die Technologie anderen Arbeitsgruppen zugänglich zu machen und ihnen die Bearbeitung von Fragestellungen zu ermöglichen, die ansonsten nur mithilfe kommerzieller Hardware adressiert werden können, veröffentlichten wir unsere Arbeiten in der Zeitschrift 3D Printing and Additive Manufacturing [1].

Ein weiteres Beispiel für ein kostengünstiges Hilfsmittel für high content imaging, das wir entwickelten, ist ein 3D gedruckter labchip für die chemische Stimulation von Zellen. Auch für diese Anwendung sind Systeme kommerziell verfügbar, die ebenso wie für die elektrische Stimulation, kostenintensiv und daher nicht für jedes Labor zugänglich sind. Die Funktionsweise ist denkbar einfach: Über einen Zugang, den man sich wie eine Infusionskanüle oder eine Glaskapillare vorstellen kann, werden Zellen kontinuierlich mit einer Lösung, die bspw. einen Rezeptoragonist oder eine Testsubstanz enthält, perfundiert. Hierbei fließt die Lösung in der
Regel gravimetrisch, d. h. der Schwerkraft folgend, aus einer Spritze durch einen Schlauch zum Zugang. Die Perfusionsdauer und Geschwindigkeit wird z. B. mithilfe eines Ventils und eines Zweiwegehahns kontrolliert. Um die Zellreaktion zu erfassen und zu analysieren, kommen typischerweise analog zu elektrischen Stimulationsexperimenten Fluoreszenzfarbstoffe und high content imaging-Systeme zum Einsatz. Ein Anwendungsbeispiel für diese Technologie ist die Analyse von Testsubstanzen zur Untersuchung einer potenziellen modulatorischen Aktivität auf Ionenkanäle. Der von uns entwickelte lab-chip hat das Format eines kleinen Deckglases $(20 \mathrm{~mm} \times 49 \mathrm{~mm}$ ) und ist mit Einlässen für Test- und Kontrolllösungen verstehen, die über einen Mikrofluidkanal in Zickzack-Form gemischt werden und in eine transparente Mikroskopie-Kammer münden, wo sich die zu analysierenden Zellen befinden. Der Boden der Mikroskopie-Kammer besteht aus einem Standard-Deckglas, das wir mit einem biokompatiblen Silikonkleber (Polydimethylsiloxan, PDMS) am Chip befestigten. Der lab-chip kann mit jedem Standard3D-Drucker in kurzer Zeit gefertigt werden und steht einige Stunden nach dem Zusammenbau für Experimente zur Verfügung. Aufgrund des einfachen Designs lässt sich die Kammer unkompliziert für andere Anwendungen (z. B. für eine größere Anzahl von Lösungen oder Mikroskopie-Kammern) modifizieren und für relativ geringe Kosten (<1 $€$ /Stück) fertigen. Ein Foto der Kammer ist in Abbildung 2A gezeigt. Die Verwendbarkeit des lab-chips bestätigten wir im Rahmen einer Machbarkeitsstudie mit Fluo-4-beladenen HEK293-Zellen, welche Hitzeschmerzbzw. Capsaicin-Rezeptoren (TRPV1) rekom- binant exprimierten. Der Rezeptor leitet infolge seiner Aktivierung Calciumionen in die Zelle, wodurch die intrazelluläre Calciumkonzentration ansteigt und die Fluoreszenzintensität des Fluoreszenzfarbstoffs zunimmt. Für die Machbarkeitsstudie transfizierten wir HEK293-Zellen zunächst in einer Standard-Kulturschale transient mit TRPV1 und inkubierten die Zellen unter Standardbedingungen für ca. 24 Stunden. Am folgenden Tag setzten wir die Zellen in den lab-chip um und kultivierten sie für weitere 24 Stunden. Vor dem Experiment beluden wir die Zellen mit dem Calciumindikator Fluo-4 und stimulierten die Zellen anschlieBend im Experiment durch Perfusion mit Kontroll- und Testlösung. Die Lösungen leiteten wir hierbei gravimetrisch aus 50-mlSpritzen, die über Schläuche mit der Kammer verbunden waren, in das Mikrofluidkanalsystem ein. Abbildung 2B zeigt Bilder einer Fluo-4-beladenen Zelle sowie den Intensitätsverlauf während einer wiederholten Stimulation mit Capsaicin (s. auch Abb. 2C) und lässt erkennen, dass die Kammer für die chemische Stimulation von Kulturzellen geeignet ist. Damit die Technologie in anderen Laboren zum Einsatz kommen kann, veröffentlichten wir diese Arbeiten in der Zeitschrift Micromachines [2].

\section{High content imaging mit}

\section{Suspensionszellen}

Im Rahmen unserer F\&E-Tätigkeiten mit rapid prototyping-Technologien und insbesondere durch die am Lehrstuhl verfügbaren 3D-Drucker initiierten wir ein Projekt, das sich der Frage widmete, ob sich ein einfacher 3D-Drucker nicht auch für den dreidimensionalen Druck von Zellen und die Biofabrikation eignet. In dem Projekt modifizierten wir einen kostengünstigen 3D-Drucker für eine Verwendung mit Zellen und setzten ihn mit rekombinanten Zellen ein, die stabil einen Fluoreszenzindikator exprimieren [3]. Als Druckmatrix zur Einbettung der Zellen für den 3D-Druck verwendeten wir u. a. eine Mischung aus Alginat und Gelatine. Diese Arbeiten brachten uns auf die Idee, Gelatine auch für chemische Stimulationsexperimente mit Suspensionszellen, z. B. Blutzellen, einzusetzen. Da Suspensionszellen nicht wie die vorhin genannten HEK293- oder MED17.11-Zellen - am Boden der Kulturschale adhärieren und bei einer Perfusion mit Kontroll- und Testlösungen Lösungen weggespült werden, ist es erforderlich, die Zellen für die mikroskopische, d. h. high con- 
tent imaging-basierte, Analyse zu immobilisieren. Gelatine ist ein biokompatibles, kostengünstiges und einfach zu verarbeitendes Proteingemisch und ist in der Vergangenheit wiederholt erfolgreich für die Biofabrikation mit verschiedenen Zelltypen zum Einsatz gekommen. Für eine Immobilisierung von Suspensionszellen und ihre anschließende fluorometrische Analyse mit high content imaging war das Material bis dato aber nicht weit verbreitet verwendet worden. Um $\mathrm{zu}$ untersuchen, ob die Gelatine-basierte Immobilisierung dazu geeignet ist, Zellen während der chemischen Stimulation, d. h. während eines Lösungswechsels oder einer Lösungszugabe, in der Kulturschale räumlich zu fixieren und gleichzeitig mikroskopisch abzulichten, führten wir proof-of-principleExperimente mit Jurkat (immortalisierte T-Lymphozyten) und THP-1-Zellen (monozytische Leukämiezellen) durch. Beide Zelllinien liegen in Suspension vor und wurden in der Vergangenheit erfolgreich für Durchflusszytometrie-basierte Experimente zur Untersuchung intrazellulärer Calciumsignale eingesetzt. Der Nachteil durchflusszytometrischer Experimente ist, dass Untersuchungen nur an großen Zellpopulationen, aber nicht auf Einzelzellebene, durchgeführt werden, sodass ohne weiterführende Studien keine Aussagen zu zellulären Subpopulationen - mit unterschiedlichen physiologischen Eigenschaften - getätigt werden können. Für unsere Machbarkeitsstudie stellten wir eine fünfprozentige Gelatinematrix in Extrazellulärmedium her und vermischten diese mit dem Pellet einer zuvor zentrifugierten Suspension aus Fluoreszenzfarbstoff-beladenen Zellen. Für die nachfolgende high content imaging-Untersuchung überführten wir die zellbeladene Gelatinematrix in eine Kulturschale, überschichteten die Matrix mit Extrazellulärmedium und zeichneten den Verlauf der Fluoreszenzintensität während der chemischen Stimulation auf. Abbildung 3A zeigt eine mikroskopische Aufnahme Gelatine-immobilisierter THP-1-Zellen. Der Intensitätsverlauf in Abbildung 3B von mit ATP bzw. Triton-X stimulierten Zellen lässt erkennen, dass nur etwa 30 Prozent aller Zellen selektiv auf die Zugabe von ATP, aber alle Zellen auf die Kontrollsubstanz Triton-X reagierten. Diese Daten zeigen nicht nur, dass unsere Methode für die Immobilisierung von Suspensionszellen und ihre high content imaging-basierte Untersuchung geeignet ist, sondern auch, dass mit diesem Ansatz auf eine einfache und kostengünstige Weise funktionelle Subpopulationen in Kulturen aus Suspensionszellen identifiziert und analysiert werden können. Die Studie veröffentlichten wir in der Zeitschrift Experimental Cell Research [4].

Diese Beispiele zeigen, dass das Anwendungsspektrum für zellbasiertes high content imaging nicht durch die apparative oder finanzielle Ausstattung eines Labors limitiert sein muss. Mithilfe von rapid prototypingTechnologien lassen sich in kurzer Zeit und mit geringem Ressourceneinsatz Hilfsmittel für high content imaging-Projekte erstellen, die zu neuen Fragestellungen führen und in spannenden Forschungsprojekten münden können.

\section{Literatur}

[1] Schneidereit D, Tschernich J, Friedrich O et al. (2018) 3D-printed reusable cell culture chamber with integrated electrodes for electrical stimulation and parallel microscopic evaluation. 3D Printing and Additive Manufacturing 5: 115-125

[3] Kahl M, Gertig M, Hoyer P et al. (2019) Ultra-low-cost 3D bioprinting: modification and application of an off-the-shelf desktop 3D-printer for biofabrication. Front Bioeng Biotechnol 7: 184
[2] Aschenbrenner D, Friedrich O, Gilbert DF (2019) 3D printed lab-on-a-chip platform for chemical stimulation and parallel analysis of ion channel function. Micromachines (Basel) 10: 548

[4] Ritter P, Bye LJ, Finol-Urdaneta RK et al. (2020) A method for high-content functional imaging of intracellular calcium responses in gelatin-immobilized non-adherent cells. Exp Cell Res 395: 112210

Funding note: Open Access funding enabled and organized by Projekt DEAL. Open Access: Dieser Artikel wird unter der Creative Commons Namensnennun 4.0 International Lizenz veröffentlicht, welche die Nutzung, Vervielfältigung, Bearbeitung, Verbreitung und Wiedergabe in jeglichem Medium und Format erlaubt, sofern Sie den/die ursprünglichen Autor(en) und die Quelle ordnungsgemäß̈ nennen, einen Link zur Creative Commons Lizenz beifügen und angeben, ob Änderungen vorgenommen wurden. Die in diesem Artikel enthaltenen Bilder und sonstiges Drittmaterial unterliegen ebenfalls der genannten Creative Commons Lizenz, sofern sich aus der Abbildungsleg
nichts anderes ergibt. Sofern das betreffende Material nicht unter der genannten Creative Commons Lizenz steht und die betreffende Handlung nicht nach gesetzlichen Vorschriften erlaubt ist, ist für die oben aufgeführten Weiterverwendungen des Materials die Einwilligung des jeweiligen Rechteinhabers einzuholen. Weitere Details zur Lizenz entnehmen Sie bitte der Rechteinhabers einzuholen. Weitere Details zur Lizenz entnehmen Sie bitte der
Lizenzinformation auf http://creativecommons.org/licenses/by/4.0/deed.de.

Korrespondenzadresse:

PD Dr. habil. Daniel F. Gilbert

Lehrstuhl für Medizinische Biotechnologie

FAU Erlangen-Nürnberg

Paul-Gordan-Straße 3

D-9 1052 Erlangen

daniel.gilbert@fau.de

www.mbt.tf.fau.de

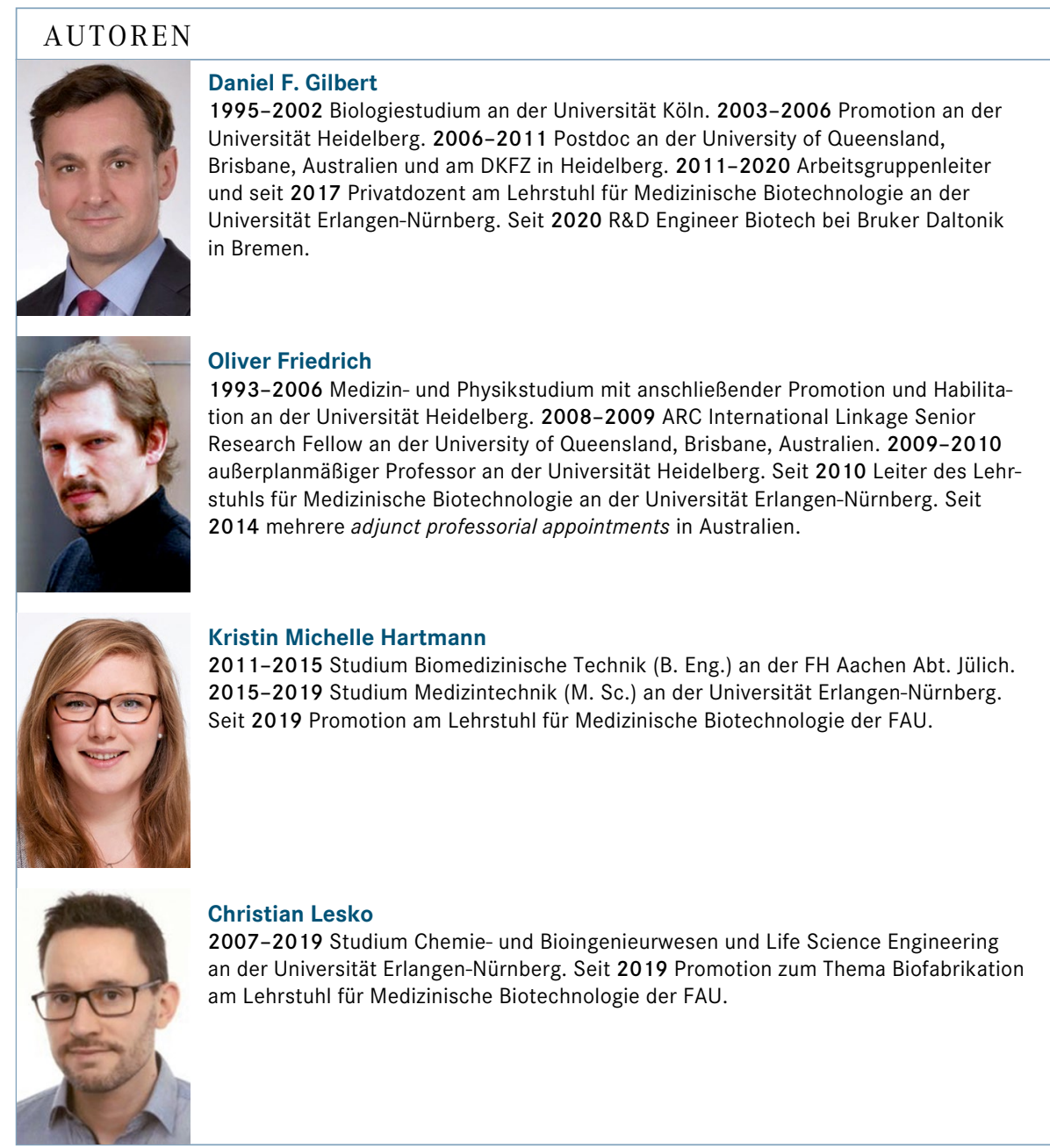

